\title{
Evolution of SSC Collider Design
}

\author{
M.J. Syphers \\ SSC Laboratory* \\ 2550 Beckleymcade Avc. \\ Dallas, TX 75237
}

\section{Abstract}

The evolution of the SSC main collider synchrotron design is discusscd. Major design changes from the 1986 Conceptual Design include the quadrupole spacing in the arcs, the injection energy, the magnet bore tube aperture, and the addition of detector bypass regions. Comments on work in progress are also presented.

\section{Introduction}

During the time since the issuance of the Conceptual Design Report (CDR) by the Central Decign Group in 1986 [1] and before the formation of the SSC Laboratory, the only major parameter change was the increase in quadrupole spacing from 96 meters to 114.25 meters associated with an increase of the phase advance per cell from 60 degrees to 90 degrees. It should not be surprising that the selection of the site brought with it a reexamination of the design incorporating the work of the past few years.

At the SSC Laboratory, effort has concentrated on tailoring the generic design to the Texas site and fleshing out the injector specifications. Additionally, SSC Laboratory personnel have felt that some steps in the direction of design conservatism should be taken. These will be described below. The material presented here represents the work of many people, as reflected by the references in the text; the present author is only attempting to summarize their work.

\section{Collider Parameters and Layout}

Injection into the Collider is the dominant factor in determining the Collider parameters. Since 16 cycles of the High Energy Booster (HEB), each of four minute duration, are required to fill the Collider, particles must be able to endure this environment for $10^{7}$ revolutions about the accelerator without degredation. The present Collider design parameters are shown in Table 1 together with the 1986

* Operated by the Universities Research Association, Inc., for the U. S. Department of Energy

U.S. Government work not protected by U.S. Copyright.
Table 1: Collider Parameters

\begin{tabular}{|l|c|c|l|}
\hline & Present & 1986 CDR & \\
\hline Injection Energy & 2.0 & 1.0 & $\mathrm{TeV}$ \\
\hline Circumference & 87120 & 82944 & $\mathrm{~m}$ \\
\hline Cell length & 90 & 96 & $\mathrm{~m}$ \\
\hline Cell phase advance & 90 & 60 & $\mathrm{deg}$ \\
\hline Dipole Coil Diameter & 50 & 40 & $\mathrm{~mm}$ \\
\hline Dipole Field & 6.60 & 6.60 & $\mathrm{~T}$ \\
\hline Max. $\beta$ in Arc & 305 & 332 & $\mathrm{~m}$ \\
\hline Max. dispersion in Arc & 1.8 & 3.9 & $\mathrm{~m}$ \\
\hline$\beta^{*}$ & 0.5 & 0.5 & $\mathrm{~m}$ \\
\hline Crossing angle & $<150$ & $<150$ & $\mu \mathrm{rad}$ \\
\hline
\end{tabular}

CDR values. The selection of basic Collider parameters is described in more detail in [2].

\subsection{Motivations for Change}

Accelerator experiments performed at Fermilab and at CERN revealed long-term particle beam loss mechanisms in the presence of guide fields with a significant admixture of nonlinearity. These observations were one of the early warning signs leading to the question of long-term stability in the SSC at injection. The causes and mechanisms of the observed diffusion are still not completely understood.

Concurrently, accelerator studies took place using the Fermilab Main Ring where the injcction environment is such that the natural dynamic aperture is on the order of the emittance of the incoming beam from the Booster synchrotron. The measured geometrical admittance of the Main Ring is more than three times the equilibrium emittance arrived at by dynamical processes. After 20 years of operation the beam hehavior in the Main Ring is not adequately understood. Though the Main Ring was not designed as a storage ring, experience with this synchrotron prompts one to look even more closely at the particle dynamics associated with the SSC at its injection energy

Complementary to the experiments mentioned above were numerical simulations. A striking feature of these simulations was that in order to account, even qualita- 
tively, for the results a rather detailed model of the synchrotron had to be developed and the tracking had to continue for orders of magnitude more turns than previously expected. Essential ingredients of the model were synchrotron oscillations, random and systematic multipole magnetic field errors, and closed orbit distortions. These results led to an extensive study of the long term behavior of particles at the injection conditions of the Collider. When this environment was first simulated for the CDR design, the resulting data indicated a dynamic aperture for $10^{5}$ turns of only $5 \mathrm{~mm}$. Simply extrapolation of the data suggests that the dynamic aperture for a particle to survive $10^{7}$ turns would be on the order of one millimeter. Though this might be a slight underestimate, nevertheless it was hard to feel comfortable with the long-term dynamic aperture of this accelerator.

\subsection{Choice of Major Parameters}

To study the parameter space of the Collider design, one needs to see the "handles" available. The three major accelcrator parameters cxplored have been the quadrupole spacing in the arcs, the injection energy, and the coil diameter of the superconducting dipole magnets.

The first order effect that the quadrupole spacing has on the accelerator performance is the determination of the beam size. For a quadrupole spacing (half cell length) $L$ and a betatron phase advance of $90^{\circ}$ per cell, the maximum amplitude function of the cell is $\hat{\beta}=3.41 \mathrm{~L}$. The dispersion function varies as the square of the quadrupole spacingand $\hat{D}=2.71 L^{2} / R$, where $R$ is the average radius of the arc. The maximum rms beam size within a cell is then

$$
\hat{\sigma}=\sqrt{\frac{\hat{\beta} \epsilon_{N}}{\pi \gamma}+\hat{D}^{2}\left(\frac{\sigma_{p}}{p}\right)^{2}}
$$

where $\epsilon_{N}$ is the rms, normalized emittance, $\gamma$ is the ratio of beam energy to the proton rest energy, and $\sigma_{p} / p$ is the rms momentum spread of the beam.

In addition to the physical extent of the beam being smaller, a shorter half cell length can result in an increase in the linear aperture. For example, the chromaticity due to systematic sextupole errors varies as $L^{3}$. 'The higher order multipoles have even higher dependences on half-cell length; for instance, the tune shift of off-momentum particles due to decapole errors $\left(b_{4}\right)$ varies as the quadrupole spacing to the seventh power.

Due to the adiabatic damping of betatron oscillations as the energy of the beam is raised, a higher injection energy for the Collider rings implies a smaller beam size and hence reduced aperture requirements. More importantly, the "persistent" current multipoles at injection will be lessened. By doubling the injection energy from $1 \mathrm{TcV}$ to 2 TeV the sextupole coefficient is cut by over a factor of two and coupled with the cell length change indicated in $\mathrm{Ta}$ ble 1 , the chromaticity of the Collider ring due to persistent current $b_{2}$ is reduced to 700 units from 3400 units. It also relaxes the alignment requirements of the dipoles enough to eliminate the need for bore tube correctors. In addition, the betatron oscillation amplitudes of the beam particles are reduced by $30 \%$ due to adiabatic damping and, in conjunction with the new half cell spacing, the beam size is reduced by $50 \%$.

The good field region of the Collider magnets can be increased by increasing the magnet coil diameter, keeping the field shape the same. If the transverse dimensions of the magnet (indicated by the coil radius $R_{o}$ ) are increased, then $b_{n} \sim 1 / R_{0}^{n}$. This scaling law applies to the systematic, or average, multipoles in the magnets which are generated by the magnet geometry or by persistent currents.

If coil placement errors are independent of the scale of the magnet, then the rms values of the multipoles will scale like $\sigma_{b_{n}} \sim 1 / R_{o}^{n+1}$. On the other hand, if placement errors scale in proportion to the size of the magnet, then $\sigma_{b_{n}} \sim 1 / R_{o}^{n}$. In either case the spread in the higher order multipoles are significantly reduced by increasing the coil radius. For comparison studies, the geometric mean of these two cases was used to perform the scaling of random multipoles, i.e., $\sigma_{b_{n}} \sim 1 / R_{o}^{n+1 / 2}$.

While many effects of various design parameters on beam performance at injection can be estimated analytically, verification with long-term particle tracking is stil] necessary. In particular, tracking is necessary for the unraveling of magnet coil diameter dependencies as well as quantitative verification of effects of the other two changes.

Simulations were initiated in late 1989 to compare the dynamic apertures of dipole magnets with $4 \mathrm{~cm}$ and $5 \mathrm{~cm}$ inner coil diameters. In an effort to speed up the analysis and to better approach the $10^{6}-10^{7}$ turn regime, a new code was developed (see, for example, [3]) in which the nonlinear kicks delivered from the dipole magnets were grouped into four regions per cell (at each major quadrupole and at the midpoints between quadrupoles) with appropriate weighting. A discussion on the choice of values can be found in [1]. The gain in dynamic aperture in going from $4 \mathrm{~cm}$ to $5 \mathrm{~cm}$ inner coil diameter is approximately a factor of 1.6 (which, coincidentally, is approximately $\left.(5 / 4)^{2}\right)$. These results, and other presentations, were reviewed by the SSC Machine Advisory Committee in December, 1989. The committee's conclusions strongly recommended a change in magnet coil diameter from $4 \mathrm{~cm}$ to $5 \mathrm{~cm}$, a program SSCL has since adopted. (See [5].)

\subsection{Overall Design}

A detailed snapshot of the SSC design may be found in the Site-Specific Conceptual Design Report (SCDR)[6]. The present Collider lattice consists of two $35 \mathrm{~km}$ arcs connected by two "cluster" regions. The clusters contain interaction regions for the major detectors and utility regions for beam injection, if system, beam scrapers, etc. Each arc contains 196 standard FODO cells, with nominally 5 dipole magnets per half cell. Space is left between the second and third dipole for mid-cell correction magnets. Ev- 


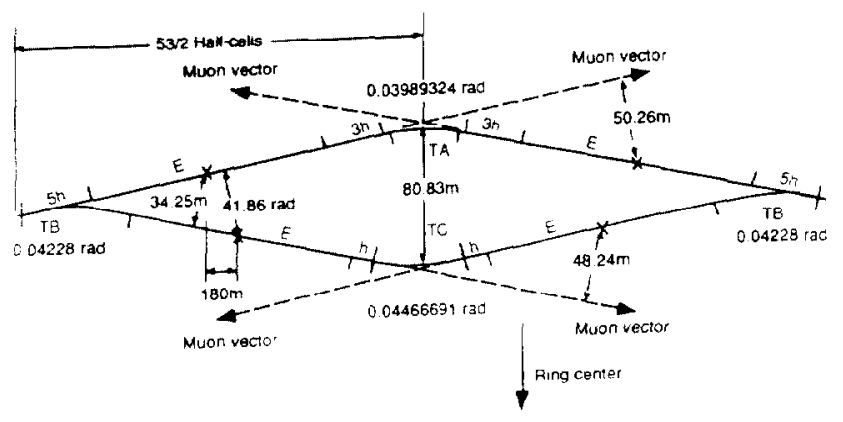

Figure 1: Interaction Region Bypass

ery six cells, a shorter dipole replaces one of the standard dipoles to allow room for cryogenic-isolation equipment.

An addition to the CDR design was the concept of "bypasses" in the cluster region to allow for future development of detectors without disturbing the ongoing physics program. The layout of a bypass is shown in Figure 1. In this "diamond" layout, the interaction regions to the outside of the ring will be built first, while future IR halls can be constructed at a later date on the inside leg of the diamond. By inserting bending magnets at the corners of the diamond during a shutdown period, one may choose to send beam through one set of detectors while $b_{i}{ }_{r}$ assing the other pair. The SCDR design has two IR's with $\beta^{*}=0.5 \mathrm{~m}$ in the West cluster, and two IR's with $\beta^{*}=10 \mathrm{~m}$ in the East cluster. Now that detector proposals are beginning to be approved, more detailed designs of these regions are just getting started.

Another change to the CDR design was to incorporate all of the injection, $r f$, and beam abort systems for both Collider rings (top and bottom) into a single utility region. These facilities were placed in the West Campus. The total utility straight section is $1.350 \mathrm{~km}$ in length and provides $500 \mathrm{~m}$ of continuous free space for removal of $20 \mathrm{TeV}$ beams toward the beam absorbers. The HEB at this location is $14 \mathrm{~m}$ directly above the lower Collider ring with its straight section aligned parallel to those of the Collider rings.

\section{Comments on Work in Progress}

Many details to the Collider design have progressed immensely since the SCDR. Much of this work is detailed elsewhere in these proceedings. Effort continues to firm up the lattice details. Small perturbations to the geometry of the Collider currently are being investigated to see whether some amount of free space can be left in the $35 \mathrm{~km}$ arcs for future use. This decision has to be made in the next month in order to progress toward start of tunnel construction. As mentioned carlicr, the IR design effort is about to begin again in earnest now that detector designs are beginning to unfold.

Energy deposition calculations are being performed to understand the environment in which superconducting magnets must survive, especially near the interaction points.[7] At the nominal luminosity, 320 Watts from the reactions will be headed in each direction toward the IR quadrupoles, which are at $4^{\circ} \mathrm{K}$ or colder. In addition, the $400 \mathrm{MJ}$ stored energy in each beam must be handled by the beam dump system at the end of each store. Monte Carlo simulations are being performed in order to design beam scraping systems[8] and beam dump systems[9].

$R \& D$ is beginning on a liner design for the Collider magnet beam tube. Two issues are at play here: intercepting the synchrotron light at a higher temperature, thereby reducing the power necessary from the cryogenic system; and improving the vacuum at the nominal temperature in lieu of desorption of hydrogen from the beam tube walls due to synchrotron radiation. The vacuum requirement is barely met by the baseline design, and increased intensity (either to meet the baseline luminosity, or to upgrade the luminosity) is pushing the limits of acceptable accelerator performance.

\section{References}

11] Conceptual Design of the Superconducting Super Collider, SSC Central Design Group, ed. J.D. Jackson, SSC-SR-2020, March 1986.

[2] Edwards, D. A., and M. J. Syphers, Selection of Basic Parameters for the Collider Rings, SSCL report SSCL-297, June 1990.

[3] Kauffmann, S., G. Bourianoff, D. Ritson, R. Steining, Tracking Studies of the Effects of Error Multipoles in the Highest Beta Focussing Quadrupolcs on SSC Perfoemance, these proceedings.

[4] Garavaglia, T., K. Kauffmann, and R. Stiening, Application of the SSCTRK Numerical Simulation Program to the Evaluation of the SSC Magnet Aperture, SSC note SSCL-265.

[5] Palmer, R., Design of Superconducting Magnets for the $S S C$, these proceedings.

[6] Site-Specific Conceptual Design Report of the Superconducting Super Collider, SSCL, ed. J. R. Sanford, SSCL-SR-1056, July, 1990.

[7] Lopez, G., and J. Shih, Quench Analysis of the Energy Depostion in the SSC Magnets and the Radiation Shielding of the Interaction Region, these proceedings.

[8] Soundranayagam, R., The SSC Collider Beam Halo Scraper System, these proceedings

[9] Parker, B., On the Design of Beam Absorbers for the $S S C$, these proceedings. 